\title{
The Effective Cloud Fraction of Broken Clouds Obtained by Multistream Radiative Transfer. Part I: Longwave Radiation
}

\author{
Hirohiko MASUnAGa \\ Earth Observation Research Center, Roppongi, Minato-ku, Tokyo, Japan \\ TERUYUKI NAKAJIMA \\ Center for Climate System Research, University of Tokyo, Komaba, Meguro-ku, Tokyo, Japan
}

(Manuscript received 3 March 2000, in final form 12 January 2001)

\begin{abstract}
The influence of broken clouds on radiative flux has provided a major source of uncertainty in radiative transfer models of the atmosphere because plane-parallel approximations are assumed in most of the current atmospheric models, where horizontal inhomogeneity cannot be adequately taken into account. In this paper, effects by cloud inhomogeneity on longwave radiation fields are investigated, using a simple model of a cloud array that consists of identical cuboids following some past studies. In contrast to past work that adopted simplified formulations of radiative transfer, multistream radiative transfer is considered to obtain the exact solutions of radiative flux, which enable us to consider semitransparent clouds as well as optically thick clouds in desirable accuracy. Applicability to semitransparent clouds is important because cirrus clouds, which are considered to play significant roles for longwave radiation, are often semitransparent to infrared radiation.

The computational results show that the empirical formula previously derived by Harshvardhan and Weinman systematically underestimates the effective cloud fraction. An alternative formula is proposed for the effective cloud fraction to supply a better fit to the exact solution of radiative flux. Furthermore, new formulas are derived to approximate the exact solutions including the dependence on the optical thickness of clouds. They are useful to convert plane-parallel flux to 3D flux passing through broken clouds, either for optically thick or thin clouds.
\end{abstract}

\section{Introduction}

Although clouds are considered to play significant roles in the earth's radiation budget, the impacts of clouds to atmospheric radiative transfer are difficult to evaluate precisely. A main uncertainty lies in the planeparallel approximations, usually assumed in calculations of atmospheric radiative transfer, because it cannot appropriately take into account the horizontal inhomogeneity (or brokenness) of clouds. The effects on the radiation fields by brokenness of clouds have been studied for more than 20 years ago by authors such as Aida (1977) for solar radiation and Harshvardhan and Weinman (1982, hereafter HW82) and Ellingson (1982) for longwave radiation. More recently, Killen and Ellingson (1994), Takara and Ellingson (1996), and Han and Ellingson (1999) have made contributions to this field. Since the exact treatment of three-dimensional radiative transfer (3D RT) requires significant computational effort, their estimations of the brokenness effects were

Corresponding author address: Dr. Hirohiko Masunaga, Earth Observation Research Center, 1-9-9 Roppongi, Minato-ku, Tokyo 1060032, Japan.

E-mail: masunaga@eorc.nasda.go.jp based on approximate methods. Aida (1977) considered the transmittance and reflectance of a cloud array as statistical average of Monte Carlo results using onecloud and nine-cloud models. HW82 proposed an empirical formula for the effective cloud fraction based on experimental data and compared it to computational results assuming the variable-azimuth two-stream (VATS) approximation. The effective cloud fraction for longwave radiation takes account of the emissions and shading by the side faces of broken cloud blocks, which enhance the emergent flux in comparison with the planeparallel flux (we hereafter call this effect the "3D effect" of clouds.) Validity of the empirical formula of HW82, however, remains to be examined by more precise calculations since it was derived to fit only six experimental data. The results of HW82, furthermore, are applicable only to optically thick clouds because both their experiments using black blocks and the VATS approximation do not yield correct solutions for optically thin clouds. The effective cloud fraction for longwave radiation was investigated on the basis of detailed studies on effect of shape and spatial distribution of black clouds (Ellingson 1982; Killen and Ellingson 1994; Han and Ellingson 1999) or of optically thick 
clouds with scattering (Takara and Ellingson 1996), while dependence on optical thickness, which possibly attenuates the geometrical effects, remained unexamined in their work. On the other hand, cirrus clouds, which are considered to play important roles for longwave radiation (Roewe and Liou 1978), are often semitransparent to infrared radiation. Therefore it is important to investigate the 3D effect of clouds with arbitrary optical thickness.

A number of sophisticated numerical schemes have been proposed to date for solving 3D RT problems in the cloudy atmosphere (e.g., Gabriel et al. 1993 and references therein). Although elaborate 3D RT schemes are powerful tools to solve a certain specific problem, they are not suitable for general circulation models (GCMs) and algorithms for satellite data analysis because of huge computational cost. In contrast, the strategy of HW82 and of this study is to supply simple formulas that are available for converting plane-parallel flux to 3D flux depending upon some parameters that characterize the cloud fields. We follow HW82 for the model of broken clouds, where an infinitely extended cloud array consists of identical cuboids. This cloud model is quite simplified compared to complicated geometry of actual clouds, but it has an advantage that the properties of cloud field can be easily characterized by well-defined parameters such as the cloud fraction and the aspect ratio.

Applicability of the effective cloud fraction may be limited because it does not adequately represent brokenness of clouds if clouds have layer-dependent cloud fraction, finite horizontal extent, and extreme internal inhomogeneity as is often the case in actual clouds. Moreover, vertically asymmetric shape of clouds would require separate effective cloud fraction for each of upward and downward fluxes. However, the effective cloud fraction is still a useful tool to approximate radiation fields in broken clouds in GCMs since elaborate treatment for 3D RT in complicated cloud structure is too time-consuming.

In contrast to HW82, we solve multistream radiative transfer to obtain the exact solutions of 3D RT, which assures reliable estimation of radiative flux regardless with optical thickness of clouds. Hence this study does not only reexamine the empirical formula of HW82 but also extends it to be applicable to clouds with any optical thickness.

In section 2 we describe the model characteristics and the equations to be solved. The computational results are presented in section 3 and discussions are made in section 4. Finally we summarize our findings in section 5 .

\section{Model descriptions}

In this section we describe our model of broken cloud fields and the formulation for calculating the radiative properties. The main target in application of our model is cirrus in the longwave window regions because cloud scattering and gas absorption are not included in the present study.

Following HW82, we adopt a regular array of cuboidal clouds for the cloud model. The cloud array is characterized by the cloud fraction, $f$, and the aspect ratio, $a$, defined by

$$
\begin{aligned}
& f=\frac{W^{2}}{(I+W)^{2}} \text { and } \\
& a=\frac{H}{W},
\end{aligned}
$$

where $W, I$, and $H$ are the width of a cloud cuboid, the interval between adjacent cloud blocks, and the cloud height, respectively. The array that consists of identical cloud blocks of $W \times W \times H$ is assumed to be isothermal and to be extended infinitely in a horizontal plane. In contrast to HW82, who assumed each of the cloud blocks is a black body, we specify arbitrary optical thickness as a free parameter in the cloud model. The vertical optical thickness inside a cloud block is denoted by $\tau_{H}$.

In the present paper, we concentrate our study on longwave radiative transfer and consider only the absorption and thermal emission without scattering. The cloud array with the source function of $S_{c}$ is irradiated by the upward flux, $\pi I_{\text {inc }}$, incident through the cloud base. In terms of the cloud temperature, $T_{c}$,

$$
S_{c}=B_{\nu}\left(T_{c}\right)
$$

for monochromatic radiation and

$$
S_{c}=\int_{\nu_{1}}^{\nu_{2}} B_{\nu}\left(T_{c}\right)
$$

for integrated radiation over the frequency range between $\nu_{1}$ and $\nu_{2}$, where $B_{v}(T)$ is the Planck function for temperature $T$. For the gray radiation, we have Eq. (3) setting $\nu_{1}=0$ and $\nu_{2}=\infty$,

$$
S_{c}=\sigma T_{c}^{4} .
$$

The incident radiation $I_{\text {inc }}$ is similarly related with the ground temperature if the atmospheric absorption between the ground and the cloud base is negligible. However, since the atmospheric absorption below the cloud can be significant for longwave radiation, the interpretation of $I_{\text {inc }}$ in terms of temperature is complicated in general. The gas absorption inside the cloud is not considered, and thus the resultant fitting formulas presented in section 3 below are applicable to the infrared window regions. Inclusion of the gas absorption inside clouds would attenuate the $3 \mathrm{D}$ effects of clouds since horizontal inhomogeneity is much less significant for gases than for clouds.

For an isothermal cloud, the upward radiance emergent through the cloud top is expressed as 


$$
\begin{aligned}
I^{\uparrow}\left(\mathbf{x}_{t}, \boldsymbol{\Omega}\right)= & I_{\text {inc }} \exp \left[-\tau\left(\mathbf{x}_{t}, \boldsymbol{\Omega}\right)\right] \\
& +S_{c}\left\{1-\exp \left[-\tau\left(\mathbf{x}_{t}, \boldsymbol{\Omega}\right)\right]\right\},
\end{aligned}
$$

where $\mathbf{x}_{t}=\left(x_{t}, y_{t}\right)$ represents the position in the top plane and $\boldsymbol{\Omega}=(\theta, \phi)$ is the unit direction vector. Cloud optical thickness along a given path parallel to $\boldsymbol{\Omega}$ measured from the point $\mathbf{x}_{t}$ is represented by $\tau\left(\mathbf{x}_{t}, \boldsymbol{\Omega}\right)$. The downward radiance emergent through the cloud base is

$$
I^{\downarrow}\left(\mathbf{x}_{b}, \boldsymbol{\Omega}\right)=S_{c}\left[1-\exp \left(-\tau\left(\mathbf{x}_{b}, \boldsymbol{\Omega}\right)\right)\right],
$$

where $\mathbf{x}_{b}=\left(x_{b}, y_{b}\right)$ is the position in the base plane. The upward (through the cloud top) and downward (through the cloud base) fluxes are calculated from Eqs. (4) and (5) as

$$
\begin{aligned}
& F^{\uparrow}=\int_{\Delta A} \frac{d x_{t} d y_{t}}{\Delta A} \int_{+2 \pi} \boldsymbol{\Omega} d \Omega I^{\uparrow}\left(\mathbf{x}_{t}, \mathbf{\Omega}\right) \quad \text { and } \\
& F^{\downarrow}=\int_{\Delta A} \frac{d x_{b} d y_{b}}{\Delta A} \int_{-2 \pi} \boldsymbol{\Omega} d \Omega I^{\downarrow}\left(\mathbf{x}_{b}, \mathbf{\Omega}\right),
\end{aligned}
$$

respectively. The spatial average of the fluxes is taken over the area $\Delta A$, which is taken as the $(W+I) \times(W$ $+I$ ) area at the cloud top or base, that is, a unit of the cloud array including both a cloud block and intercloud sky. The integrals over solid angle range over the upper hemisphere in Eq. (6) and the lower hemisphere in Eq. (7), that is,

$$
\begin{aligned}
& \int_{+2 \pi} \boldsymbol{\Omega} d \boldsymbol{\Omega}=\int_{0}^{1} \mu d \mu \int_{0}^{2 \pi} d \phi \\
& \int_{-2 \pi} \boldsymbol{\Omega} d \boldsymbol{\Omega}=\int_{-1}^{0} \mu d \mu \int_{0}^{2 \pi} d \phi
\end{aligned}
$$

where $\mu$ is cosine of the zenith angle.

In numerical calculations, Eqs. (6) and (7) are discretized as

$$
\begin{aligned}
F^{\uparrow} & =\left(\sum_{n=1}^{N} \delta A_{n} \times \sum_{j=1}^{J} w_{j} \cos \theta_{j} I_{n j}^{\uparrow}\right) / \Delta A \quad \text { and } \\
F^{\downarrow} & =\left(\sum_{n=1}^{N} \delta A_{n} \times \sum_{j=1}^{J} w_{j} \cos \theta_{j} I_{n j}^{\downarrow}\right) / \Delta A,
\end{aligned}
$$

where $N$ is the total number of discretized cells inside $\Delta A, \delta A_{n}$ the area of the $n$-th cell, $J$ the total number of the discretized direction angle, $w_{j}$ the weight for the numerical integration over solid angle, and $\theta_{j}$ the zenith angle. For numerical integration of $I_{n i}^{\uparrow}$ and $I_{n j}^{\downarrow^{j}}$ according to Eqs. (4) and (5), the integral path is traced in the $i$ th direction from the cloud top to the base (or from base to top) under the cyclic boundary condition on the horizontal boundaries. Note that upward radiance incident through the side faces and the photon exchange between clouds are correctly taken into account in computations due to the cyclic boundary condition. In our calculations, $N$ is $50 \times 50$ and $w_{j}$ is determined according to a level symmetric $S_{8}$ quadrature formula, that is, $J=40$ within a hemisphere (Lewis and Miller 1984).

Since our interest in this paper is not to obtain the absolute values of radiative flux but is limited on the geometrical effects caused by broken clouds, the computational flux presented in this paper is normalized by the plane-parallel flux, $F_{\mathrm{PP}}^{\uparrow}$ or $F_{\mathrm{PP}}^{\downarrow}$. This strategy has the advantage that the results do not suffer from the uncertainties in our knowledge on the optical properties of clouds because information of the extinction coefficients of water droplets and ice crystals tends to be canceled out in the ratios of $F^{\uparrow} / F_{\mathrm{PP}}^{\uparrow}$ and $F^{\downarrow} / F_{\mathrm{PP}}^{\downarrow}$. The plane-parallel flux for an isothermal cloud sheet is

$$
\begin{aligned}
& F_{\mathrm{PP}}^{\uparrow}=\pi S_{c}+2 \pi\left(I_{\mathrm{inc}}-S_{c}\right) E_{3}\left(\tau_{\mathrm{PP}}\right), \\
& F_{\mathrm{PP}}^{\downarrow}=\pi S_{c}\left[1-2 E_{3}\left(\tau_{\mathrm{PP}}\right)\right],
\end{aligned}
$$

where

$$
E_{n}(y)=\int_{1}^{\infty} \frac{d x}{x^{n}} \exp (-x y)
$$

is the exponential integral and $\tau_{\mathrm{PP}}$ is the vertical optical thickness inside the plane-parallel cloud sheet. The height of the plane-parallel cloud is equal to that of a block of the broken cloud array, $H$.

We consider two different cases for determining $\tau_{\mathrm{PP}}$, that is, $\tau_{\mathrm{PP}}=\tau_{H}$ and $\tau_{\mathrm{PP}}=f \tau_{H}$. The first case $\left(\tau_{\mathrm{PP}}=\right.$ $\tau_{H}$ ) provides a plane-parallel cloud sheet that is filled with cloud material of the same vertical optical thickness as $\tau_{H}$, while another plane-parallel cloud sheet consists of spatially averaged cloud material conserving its total amount. For the sake of convenience, we call the former case as the maximum plane-parallel case and the latter the average plane-parallel case. These cases represent two different ways in which the broken cloud fields are related to the plane-parallel approximation. The maximum plane-parallel model has the same liquid water path (LWP) as a block of the broken cloud system but a larger mass of water/ice in total, whereas the average plane-parallel model contains the equivalent total mass of water/ice but has a lower LWP. Actual plane-parallel model would lie between these two limiting cases when one applies the plane-parallel approximation to radiative transfer models used in GCMs or satellite data analysis. We will comment on the applicability of these two plane-parallel models in section $3 \mathrm{e}$.

Although we use only the spatially averaged flux [Eqs. (6) and (7)] in this work, it may be helpful to show the spatial distribution of the flux before averaging to demonstrate how the broken cloud field influences radiative flux. The spatial distribution of the upward and downward fluxes normalized by the plane-parallel value (in the maximum plane-parallel model) is drawn in Fig. 1. A unit of the cloud array is presented that contains a cloud block $(0 \leq x, y \leq W)$ and clear-sky region (elsewhere). In this case, we assume $f=0.25$ (and therefore $W=I), a=1, \tau_{H}=10$, and $S_{c} / I_{\mathrm{inc}}=0.5$. The upward flux shows a complicated spatial structure 


\section{Optical Depth $=10$}

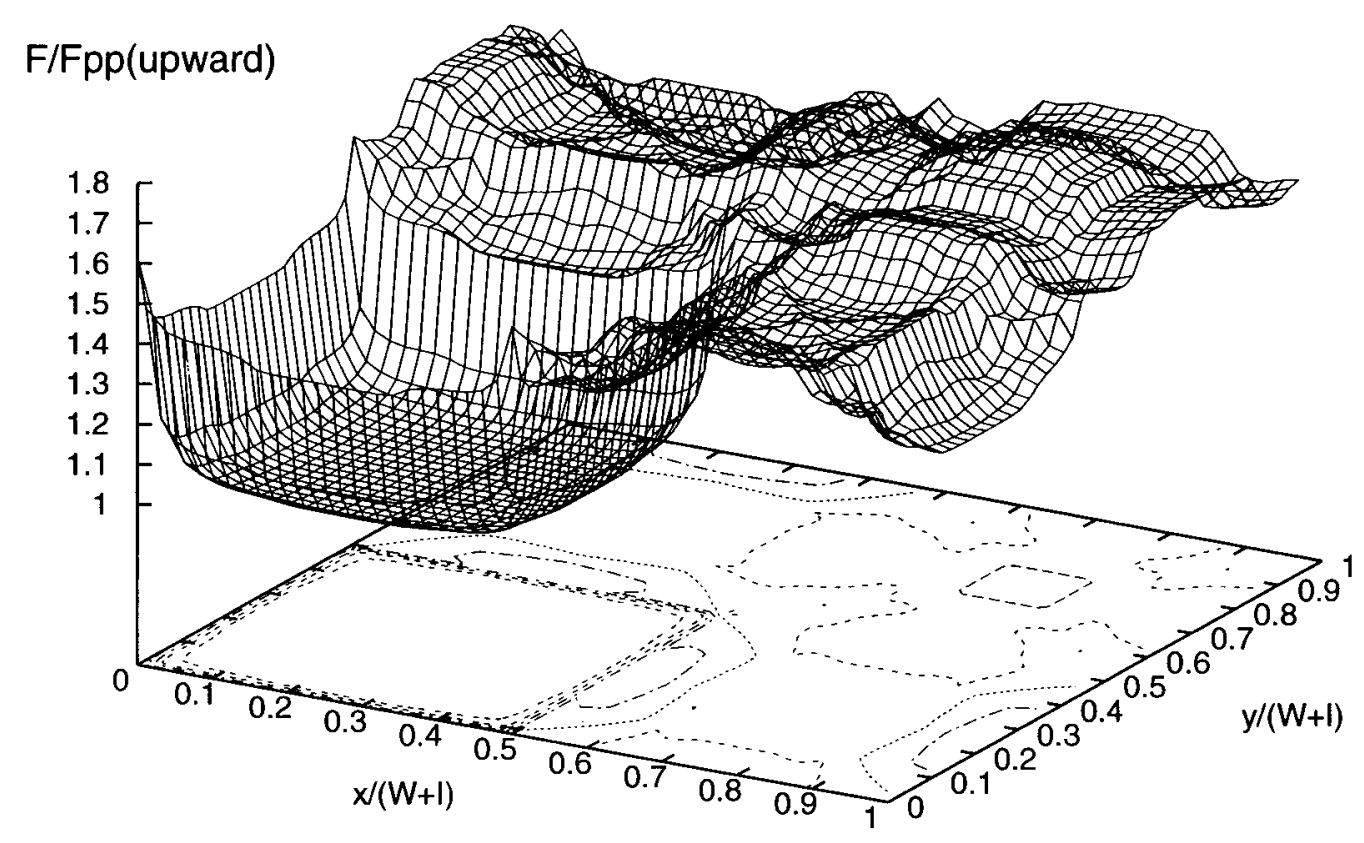

F/Fpp(downward)

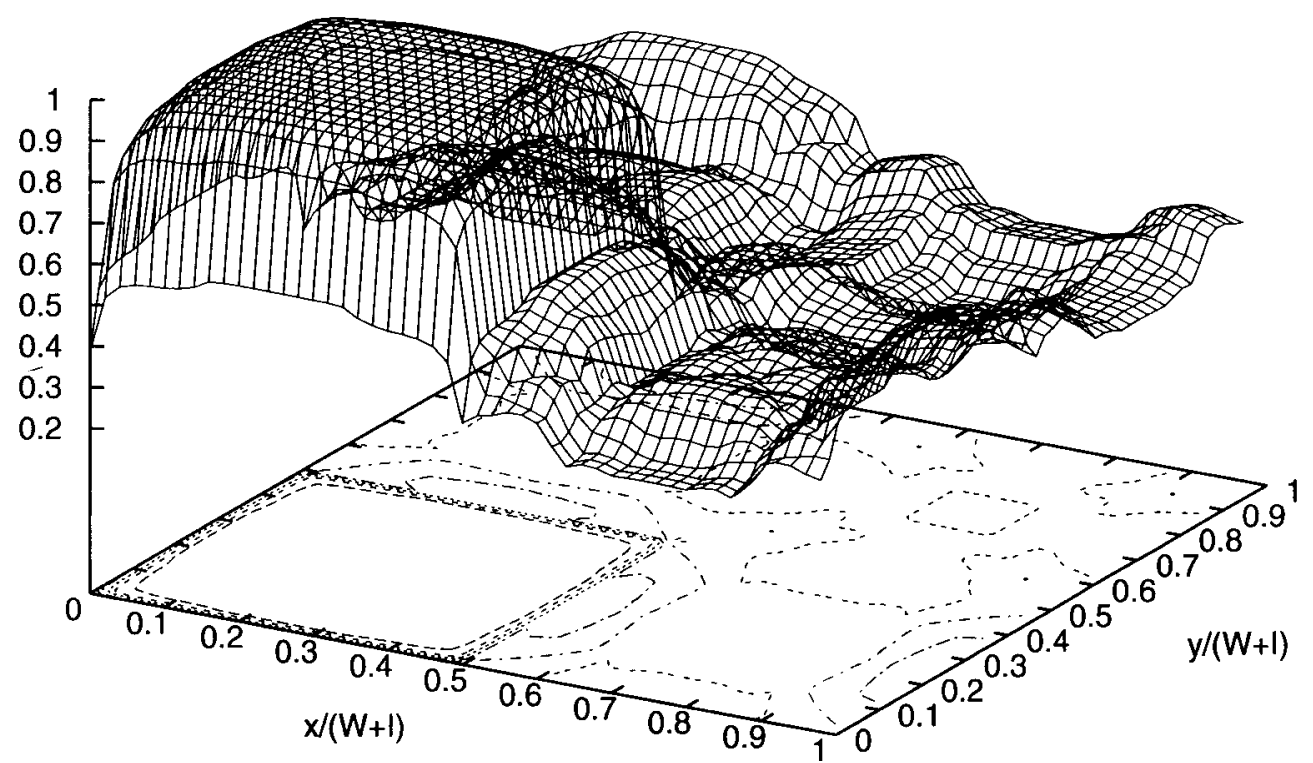

FIG. 1. Spatial distribution of radiative flux for $\tau_{H}=10$. Upward flux emergent through the cloud top (top) and downward flux through the cloud base (bottom). 


\section{Optical Depth $=1$}

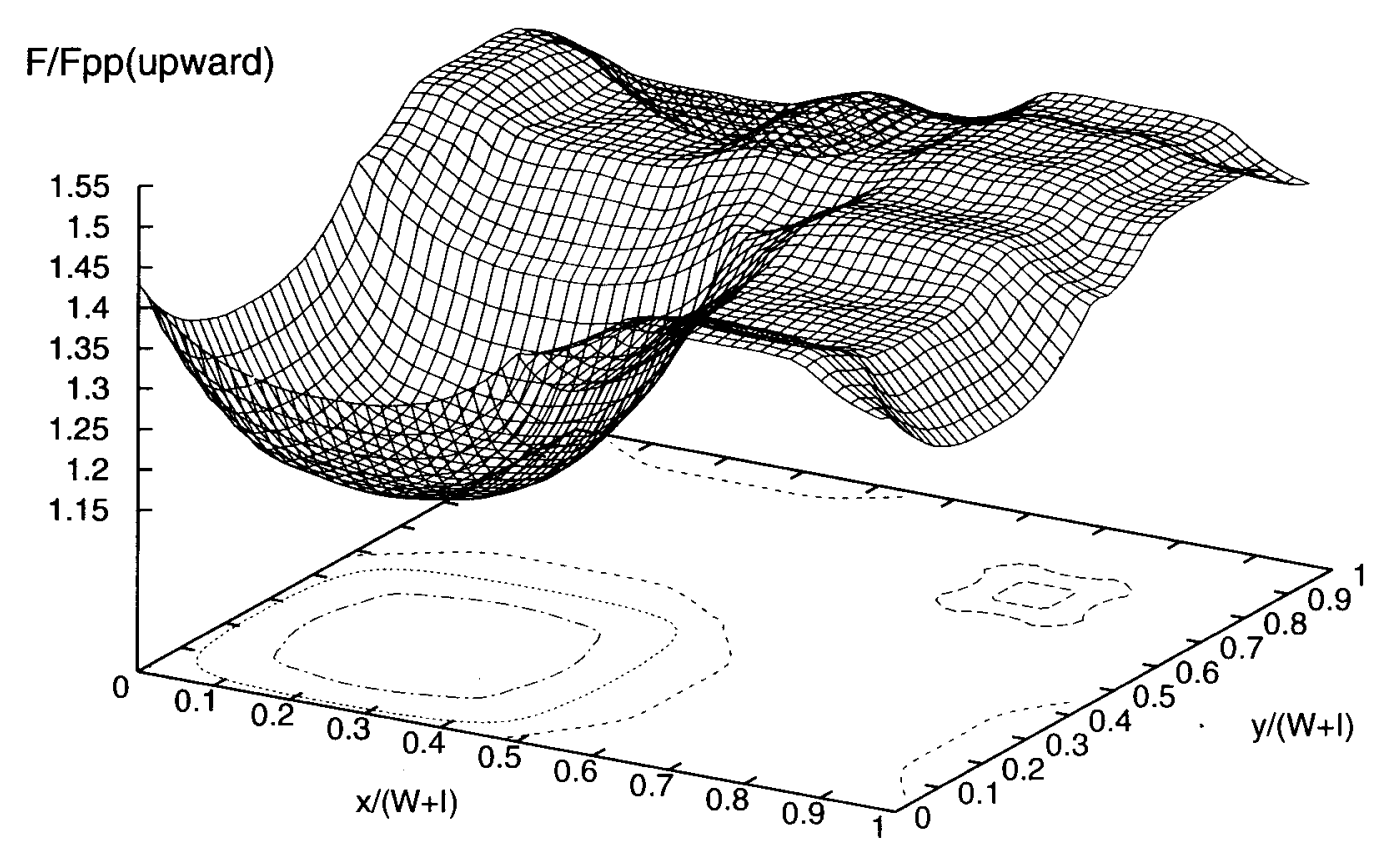

\section{F/Fpp(downward)}

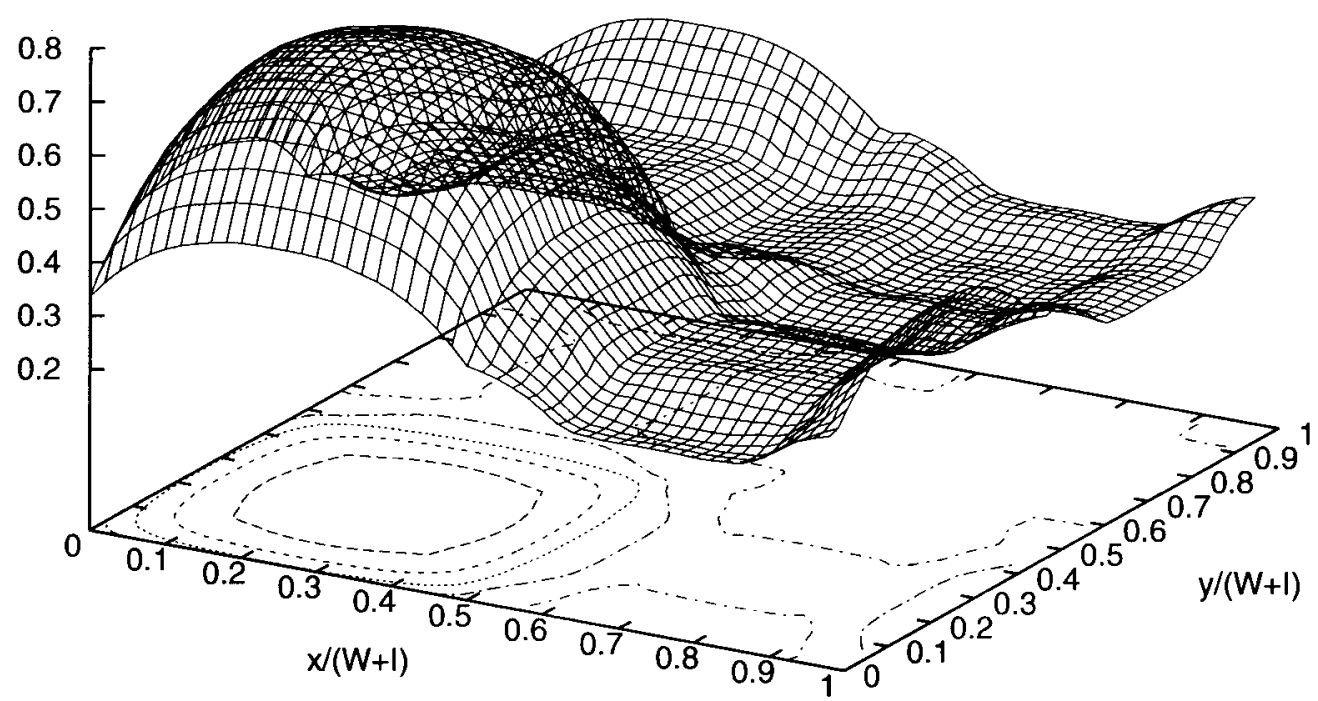

FIG. 2. Spatial distribution of radiative flux for $\tau_{H}=1$. Upward flux emergent through the cloud top (top) and downward flux through the cloud base (bottom).

as a result of combined effects of the illumination by the ground radiation and the emission from the cloud surfaces, partially shaded by the cloud itself. While the upward flux is substantially enhanced beyond the plane- parallel value $(\approx 1)$ in the clear-sky region since $I_{\text {inc }}>$ $S_{c}$, the flux just above a cloud block is very close to the plane-parallel value because of shading by the clouds. In the downward flux, an external component 

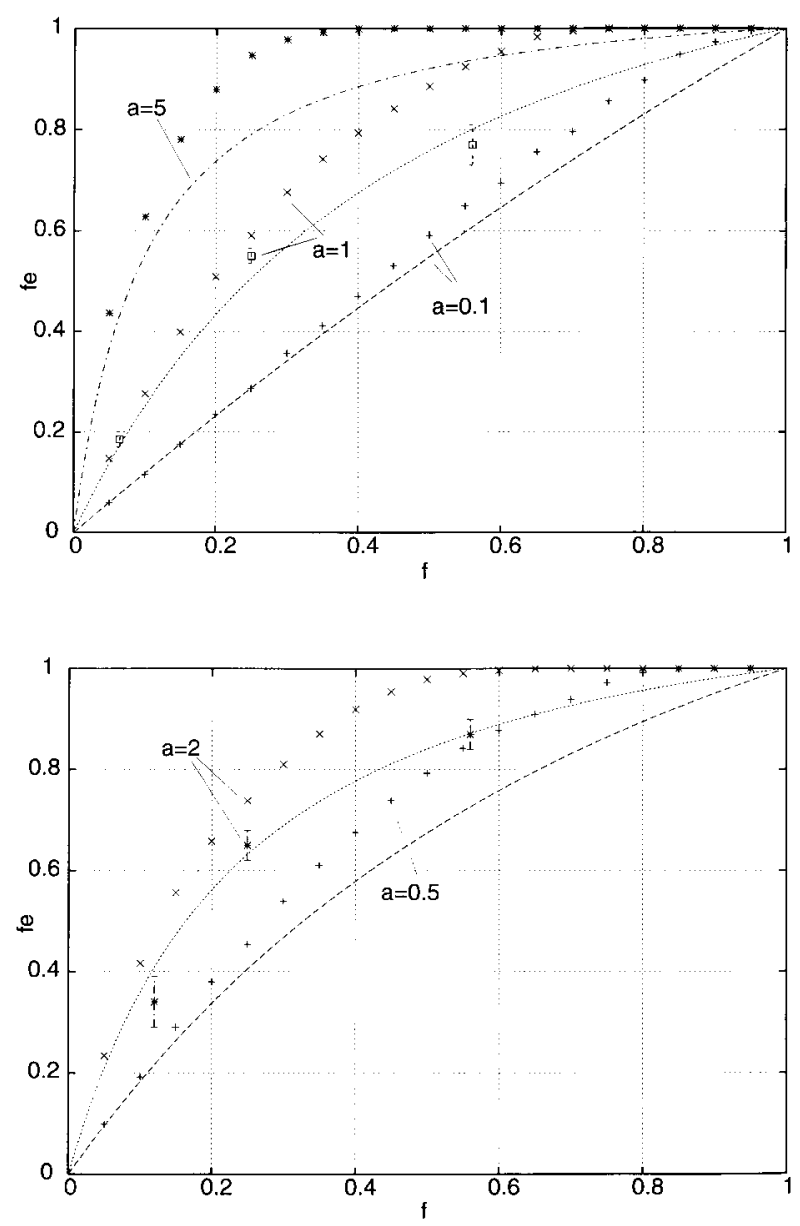

FIG. 3. Effective cloud fraction for $\tau_{H}=50$ with the empirical formula by HW82 [Eq. (19)] is shown (line). Symbols without error bars denote the exact solution given by numerical calculations in the present study, while the other symbols with error bars show the experimental data obtained by HW82.

such as the ground radiation is not included and therefore the cloud acts only as an emitter, not as an absorber, which produces an upside-down structure of the upward flux. Figure 2 is the same as Fig. 1 except that $\tau_{H}$ equals 1 instead of 10 . The spatial structure is much more smoothed here since an optically thin cloud no longer absorbs nor emits effectively.

We define the effective cloud fraction as follows:

$$
f_{e}=\frac{F^{\downarrow}}{F_{\mathrm{PP}}^{\downarrow}} .
$$

Equation (12) is identical to the definition by HW82 when the cloud is optically thick enough to be a blackbody.

Combining Eqs. (5), (7), (11) and (12), one finds

$$
f_{e}=\frac{1-2 \Lambda}{1-2 E_{3}\left(\tau_{\mathrm{PP}}\right)},
$$

where

$$
\Lambda=\frac{1}{2 \pi} \int_{\Delta A} \frac{d A}{\Delta A} \int_{-2 \pi} \boldsymbol{\Omega} d \mathbf{\Omega} \exp \left(-\tau\left(\mathbf{x}_{b}, \boldsymbol{\Omega}\right)\right)
$$

Equation (13) shows that $f_{e}$ depends only on geometry of the cloud field and on optical thickness, independently of $S_{c}$. Under given $f_{e}$, the downward flux is related with plane-parallel flux through a rearranged form of Eq. (12), that is,

$$
F^{\downarrow}=f_{e} F_{\mathrm{PP}}^{\downarrow},
$$

and the upward flux is through

$$
F^{\uparrow}=f_{e} F_{\mathrm{PP}}^{\uparrow}+\left(1-f_{e}\right) \pi I_{\mathrm{inc}},
$$

derived from Eqs. (4)-(7) and (10)-(13). Equation (16) reduces to Eq. (10) of HW82 when the cloud is optically thick. Equations (15) and (16) are equivalent to Eq. (1) in Han and Ellingson (1999) if downward clear-sky flux multiplied by $\left(1-f_{e}\right)$ is added to the rhs of Eq. (15). Downward clear-sky flux is necessary at the surface where water vapor emission is not negligible in clearsky flux, but is omitted here for brevity. However, the effective cloud fraction defined by Eq. (12) is readily applied to estimate downward flux in the presence of the clear-sky flux using a generalized formulation such as Eq. (1) in Han and Ellingson (1999).

Partially covered cloud field in GCMs or in algorithms for observational data analysis may be taken into account according to

$$
\begin{aligned}
& F^{\downarrow}=f F_{\mathrm{PP}}^{\downarrow} \quad \text { and } \\
& F^{\uparrow}=f F_{\mathrm{PP}}^{\uparrow}+(1-f) \pi I_{\mathrm{inc}},
\end{aligned}
$$

where the $3 \mathrm{D}$ effect of clouds is not considered. Once obtaining $f_{e}$ as a function of parameters characterizing the brokenness of clouds, one can incorporate the 3D effect of clouds into plane-parallel models through Eqs. (15) and (16) instead of Eqs. (17) and (18) with no additional effort for computation.

In the subsequent section, we present the numerical solutions for $f_{e}$ and provide some formulas approximating the solutions. These formulas, combined with Eqs. (15) and (16), are intended to be used as correction factors, with which the $3 \mathrm{D}$ effect of clouds is approximately taken into account in plane-parallel calculations performed in GCMs and remote sensing data analysis.

\section{Computational results}

In this section, we show the computed radiative flux for a broken cloud array based on the model described in section 2. According to HW82, the effective cloud fraction $f_{e}$ defined by Eq. (12) is presented as a function of the (true) cloud fraction $f$ given by Eq. (1). In addition to the exact solutions for $f_{e}$ obtained by multistream calculations for radiative transfer, we present some approximate formulas for more useful application of $f_{e}$.

We have two different forms of $f_{e}$ in Eq. (12) de- 

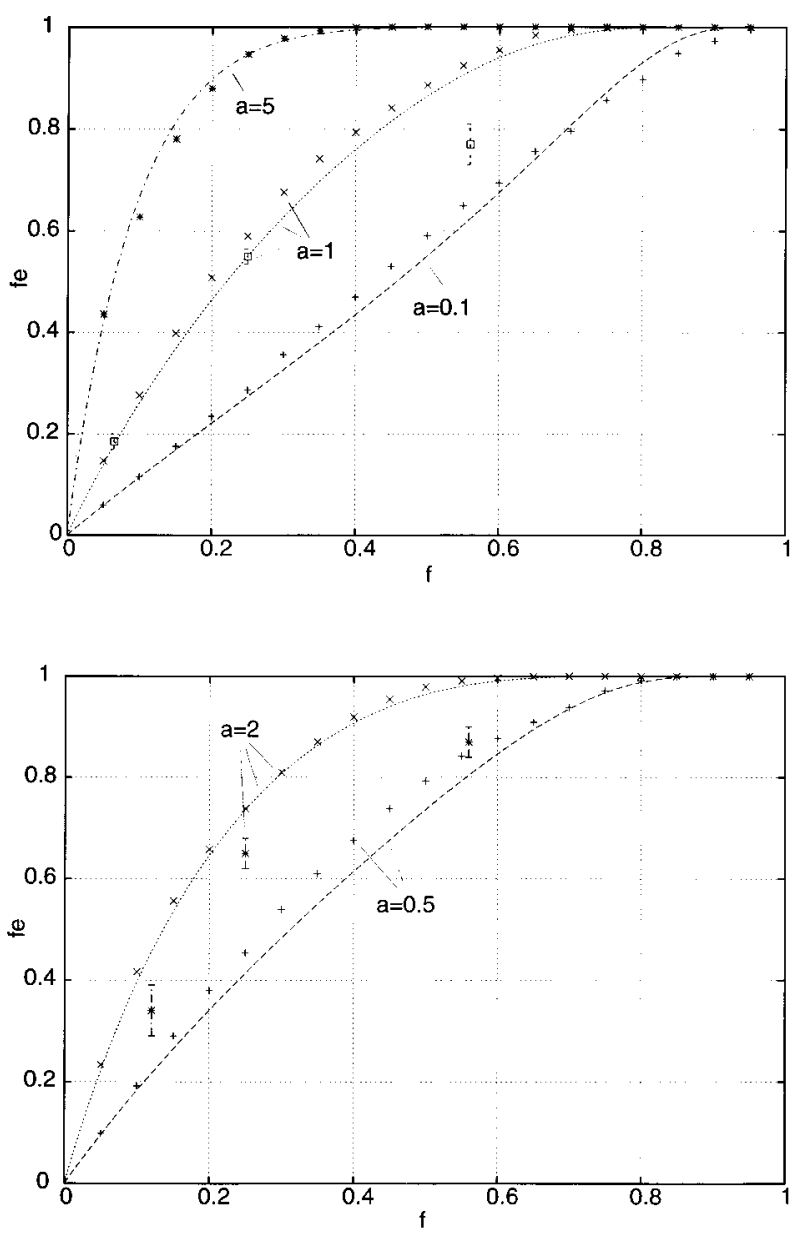

FIG. 4. Effective cloud fraction for $\tau_{H}=50$ with the approximate formula newly proposed by Eq. (20) is shown (line). Symbols with and without error bars are the same as in Fig. 3.

pending upon the definition of $F_{\mathrm{PP}}^{\uparrow}$, that is, the maximum plane-parallel or the average plane-parallel case. In the following sections, we first present the optically thick case on the basis of the maximum plane-parallel model to compare our results with HW82. Second, the dependence on optical thickness is examined for the maximum plane-parallel case, and finally $f_{e}$ for the average planeparallel case is shown.

\section{a. Optically thick case in comparison with HW82}

First, we consider the cloud array of $\tau_{H}=50$, which is so optically thick that the cloud system can be considered as blackbody as was the case in HW82. Figure 3 depicts $f_{e}$ as a function of $f$ for $a=0.1,0.5,1,2$, and 5. Besides our computational results (plots without error bars), Fig. 3 shows the experimental results by HW82 (plots with error bars) and HW82's formula for blackbody clouds (lines) defined as

$$
f_{e}^{\mathrm{BB}}=\frac{[1+2 a(1+0.15 f)] f}{1+2 a f(1+0.15 f)} .
$$

One can see that $f_{e}$ as a function of $f$ traces convex curves due to the $3 \mathrm{D}$ effect of clouds. Although the empirical formula of Eq. (19) and the exact solutions by our calculations exhibit a qualitatively similar trend in Fig. 3, the empirical formula of Eq. (19) provides systematically smaller values of $f_{e}$ than the exact solutions by our calculations. The discrepancy is as much as $\sim 10 \%$ in $f_{e}$ for large $a$.

As a more precise function to fit the exact solution, we propose another approximate formula on $f_{e}$ for blackbody clouds as follows:

$$
f_{e}^{\mathrm{BB}}=1-\exp \left[-\frac{(1+2 a) f}{1-f^{2}}\right],
$$

which is delineated in Fig. 4 with the same plots as in Fig. 3. Equation (20) provides a better fit to the exact solution than Eq. (19) shown in Fig. 3. In comparison with the experimental data by HW82, Eq. (20) successfully reproduces two of the six data points but fails in the other four, all of which are considerably smaller in $f_{e}$ than predicted by Eq. (20). The reason is unclear why the experimental data do not agree with our exact solution. Nevertheless, it seems reasonable that the two experimental data in excellent agreement with the exact solutions have shorter error bars, thus higher accuracy, than the other four.

In the limit of low cloud fraction $(f \rightarrow 0), f_{e}^{\mathrm{BB}}$ should be $(1+2 a) f$ because the radiation emitted from cloud surfaces is escaped away without disturbed by the other clouds, where $f_{e}^{\mathrm{BB}} / f$ equals the ratio of the total emitting area of a cloud block to that of a planiform cloud piece, that is,

$$
\frac{f_{e}^{\mathrm{BB}}}{f}=\frac{W^{2}+2 W H}{W^{2}}=1+2 a,
$$

as also noted by HW82. This condition is satisfied in Eq. (21) for $f \ll 1$ as well as in Eq. (19). Another condition is required that $f_{e}^{\mathrm{BB}}$ should accord with $f$ for $a=0$, which is not satisfied in Eq. (20) in the exact sense. This, however, causes no practical inconvenience since the error is small.

\section{b. Effective cloud fraction for general optical thickness based on the maximum plane-parallel model}

HW82 derived Eq. (19) assuming the cloud array is black, and then applied it to a more realistic cloud model that is partially transparent using the VATS approximation (Harshvardhan et al. 1981). The VATS approximation is, however, not exact for optically thin clouds as also implied by HW82. In contrast, the multistream treatment of radiative transfer in this paper yields accurate solutions for any optical thickness. We extend the black $\left(\tau_{H}=50\right)$ cloud model considered above to more general models with variable optical thickness. We employ the maximum plane-parallel model for calcu- 

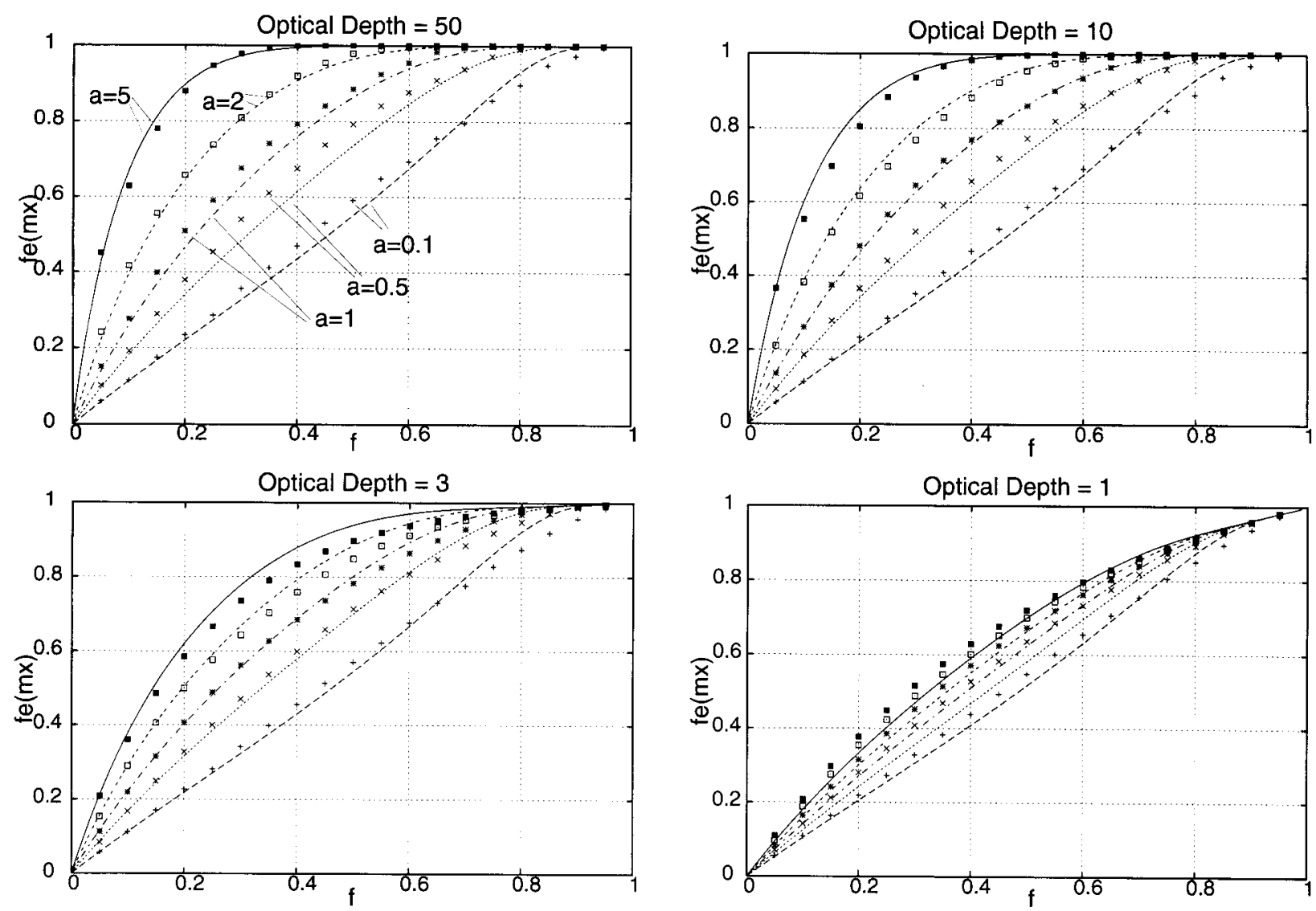

FIG. 5. Effective cloud fraction for $\tau_{H}=10,3,1$ as well as $\tau_{H}=50$ with the approximate formula given by Eq. (22) is shown (line). Symbols denote the exact solutions as in Fig. 3. The maximum plane-parallel model is used for the normalizing flux.

lating the normalizing flux, that is, $\tau_{\mathrm{PP}}=\tau_{H}$ in Eq. (11), throughout the present section.

Figure 5 presents the $f_{e}$ versus $f$ diagrams for $\tau_{H}=$ 10,3 , and 1 as well as $\tau_{H}=50$. The figure shows that a smaller optical thickness results in smaller excess of $f_{e}^{\mathrm{BB}}$ over $f$. In other words, the 3D effect of semitransparent clouds causes less significant influences on $f_{e}$ compared to opaque clouds. This tendency is easily understood because semitransparent clouds do not effectively shade the surrounding radiation, which attenuates the 3D effect on the radiation fields. We will, however, show in section $3 \mathrm{~d}$ that the result is totally different when the average plane-parallel model is adopted instead of the maximum plane-parallel model for calculating the normalizing flux.

The formula of $f_{e}$ for general optical thickness on the basis of the maximum plane-parallel case is proposed as

$$
f_{e}^{\mathrm{mx}}=c_{1}\left[1-\exp \left(-\frac{\left(1+2 c_{2} a\right) f}{1-f^{2}}\right)\right]+\left(1-c_{1}\right) f,
$$

where

$$
c_{1}=1-\exp \left(-\tau_{H}\right), \quad c_{2}=1-\exp \left(-\frac{\tau_{H}}{1+a}\right)
$$

In the optically thick limit, $c_{1}$ and $c_{2}$ become unity and then $f_{e}^{\mathrm{mx}}$ is identical to $f_{e}^{\mathrm{BB}}$. Equation (22) is delineated in Fig. 5 for each $\tau_{H}$ and each $a$ in addition to the exact solutions indicated by plots. Accordance of the approximate formula with the exact solutions is not always excellent in particular for small $\tau_{H}$, but the error would not be serious for practical use.

\section{c. Dependence on the normalizing plane-parallel models}

We discuss here the upward and downward fluxes emergent from the broken cloud fields for each of the maximum plane-parallel and average plane-parallel cases. Figure 6 shows $F^{\uparrow} / F_{\mathrm{PP}}^{\uparrow}$ and $F^{\downarrow} / F_{\mathrm{PP}}^{\downarrow}$ calculated by Eq. (8) with Eq. (10), and by Eq. (9) with Eq. (11), respectively, as a function of $\tau_{H}$ using the maximum planeparallel model. Two cases for the cloud fraction, $f=$ 0.25 and 0.75 , are considered. We assume $S_{c} / I_{\text {inc }}=0.5$ for computing the upward flux. (The downward flux is independent of this value.) The normalized downward flux, $F^{\downarrow} / F_{\mathrm{PP}}^{\downarrow}$, is equivalent to $f_{e}$ by its definition.

The downward flux increases with $\tau_{H}$ but saturates in the optically thick limit, where the saturated value cor- 

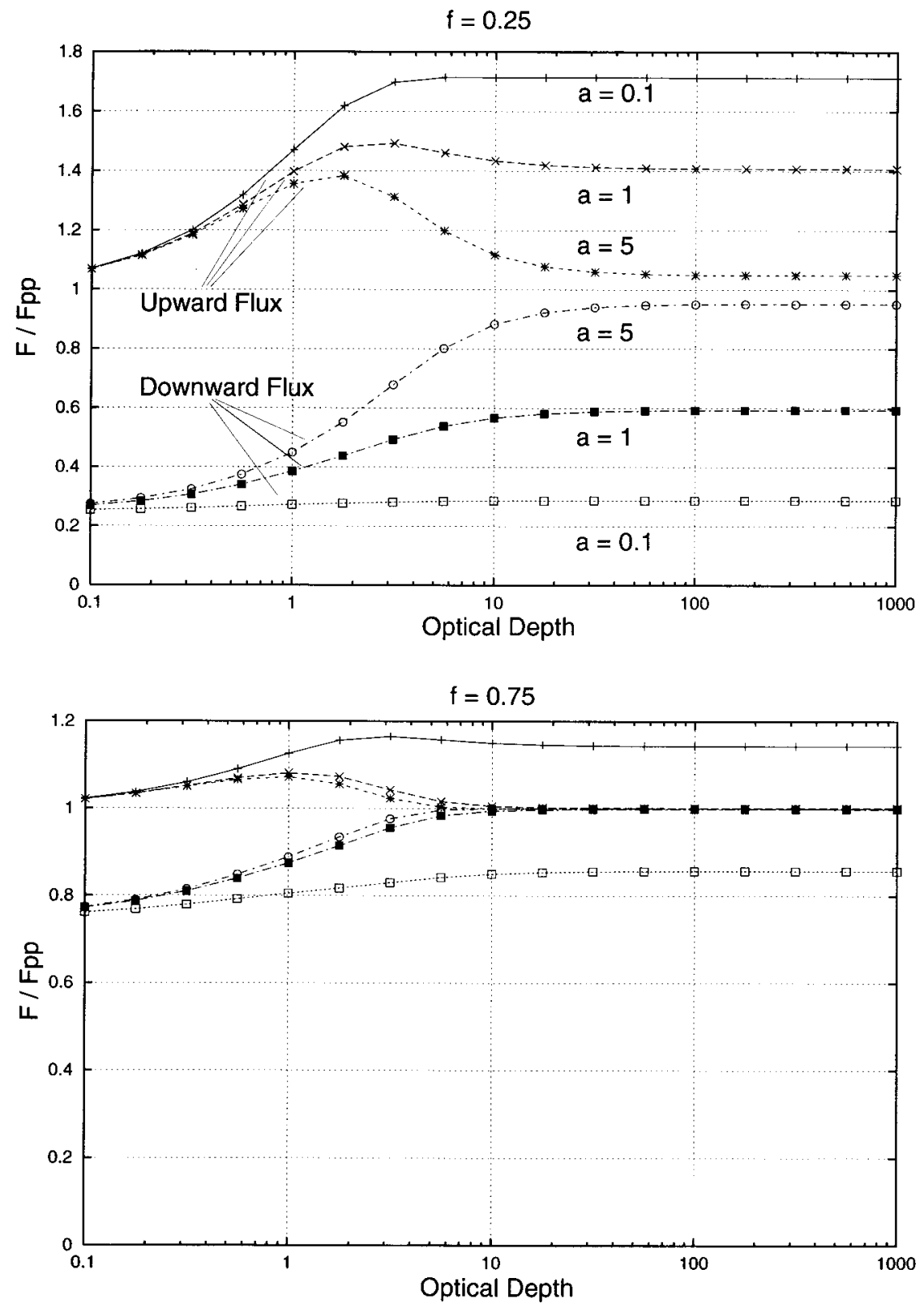

FIG. 6. The upward flux emergent through the cloud top and the downward flux through the base for the maximum plane-parallel model, i.e., $\tau_{\mathrm{PP}}=\tau_{H}$.

responds to $f_{e}^{\mathrm{BB}}$. The increase of $F^{\downarrow}$ with $\tau_{H}$ for moderately transparent clouds is more rapid for larger $a$, where the the side faces of clouds enhance their relative importance. The optically thin limit of $F^{\downarrow} / F_{\text {PP }}^{\downarrow}$ converges to $f$ for all values of $a$. The upward and downward fluxes satisfy the condition of $\left(F^{\uparrow} / F_{\mathrm{PP}}^{\uparrow}+F^{\downarrow} / F_{\mathrm{PP}}^{\downarrow}\right) / 2=$ 1 for opaque clouds, because the side faces behave as emitters for the downward flux while as absorbers for the upward flux, and these effects compensate for each other. However, this condition breaks down for semitransparent clouds, where the upward flux tends to ap- proach the plane-parallel value in the optically thin limit independently of $a$. Semitransparent clouds are not effective absorbers and hence the brokenness of clouds no longer has significant effect on the radiation fields.

Figure 7 illustrates the emergent flux similarly to Fig. 6 but using the average plane-parallel model. A most striking difference from the maximum plane-parallel case is that $F^{\downarrow} / F_{\mathrm{PP}}^{\downarrow}$, as well as $F^{\uparrow} / F_{\mathrm{PP}}^{\uparrow}$, approaches to unity in the optically thin limit. This trend results from the fact that the emission from semitransparent clouds directly reflects the total amount of cloud material, and 
therefore $F^{\downarrow}$ is close to $F_{\mathrm{PP}}^{\downarrow}$ for $\tau_{H} \ll 1$ if the total mass of water/ice is common as is the case in the average plane-parallel case. On the other hand, the behavior in the optically thick limit shows no difference for the both cases because the both plane-parallel clouds fall to an equivalent cloud sheet of blackbody when $\tau_{H}$ is sufficiently large.

\section{d. Effective cloud fraction for general optical thickness based on the average plane-parallel model}

In this section we show $f_{e}$ as a function of $f$ for the average plane-parallel case. Figure 8 draws $f_{e}$ as a function of $f$ for $\tau_{H}=50,10,3$, and 1, similar to Fig. 5 .

As expected, an opaque cloud $\left(\tau_{H}=50\right)$ yields essentially the same dependence of $f_{e}$ on $f$, while semitransparent clouds give totally different features. In contrast to the maximum plane-parallel case, $f_{e}$ does not converge to zero when $f \rightarrow 0$ and instead $f_{e}$ tends to increase up to unity as $\tau_{H}$ decrease. This tendency directly reflects the trend of $F^{\downarrow} / F_{\mathrm{PP}}^{\downarrow}$ (or $f_{e}$ ) $\rightarrow 1$ for $\tau_{H} \rightarrow 0$ in Fig. 7 as mentioned in section $3 \mathrm{c}$. The approximate formula of $f_{e}$ for the average planeparallel model, drawn by lines in Fig. 8, is given by

$$
f_{e}^{\mathrm{av}}=c\left\{1-\exp \left[-\frac{(1+2 c a) f}{1-f^{2}}\right]\right\}+1-c,
$$

where

$$
c=1-\exp \left(-\frac{\tau_{H}}{1+2 a}\right)
$$

Equation (23) is equivalent to Eq. (20) for $\tau_{H} \rightarrow \infty$.

\section{e. Applications}

In actual applications, either of the maximum or average plane-parallel case, or their combination, should be chosen depending on purpose. When one applies $f_{e}$ to correction of flux in fields of view covered partially with clouds in infrared or microwave remote sensors, the average plane-parallel case would be appropriate because the observed flux is smoothed out within a field of view. In contrast, the maximum plane-parallel case might be preferable in GCMs in case that a plane-parallel cloud is assumed within a grid cell instead of broken clouds of the same liquid water density.

As shown above, $f_{e}^{\mathrm{mx}}$ and $f_{e}^{\mathrm{av}}$ are identical for optically thick clouds, but they exhibit essentially different behaviors for semitransparent clouds. Even if $\tau_{H}$ is as much as 10, $f_{e}^{\mathrm{mx}}$ (in Fig. 5) is distinct from $f_{e}^{\mathrm{av}}$ (in Fig. 8) for high aspect ratios, $a=2$ and 5, for which the horizontal optical thickness is not large enough. Considering that clouds typically have optical thickness of $\sim 10$ or less, one finds that the assumption that clouds are black is generally invalid and the normalizing plane- parallel model for determining $f_{e}$ should be chosen appropriately corresponding to the situation.

The independent pixel approximation (IPA) is known to be a useful tool for calculating radiation flux in the presence of horizontally inhomogeneous clouds. In the IPA the cloud inhomogeneity is represented by a set of plane-parallel columns with different optical properties, where horizontal radiative interactions are neglected. Although the IPA reduces computational time for 3D $\mathrm{RT}$, it can fail to produce some essential physical processes where the horizontal photon transport is important, such as the radiative smoothing in fractal clouds (Marshak et al. 1995). Derivation of the effective cloud fraction requires a precise calculation of the horizontal energy transfer by radiation since it involves the emission and absorption by the side faces of broken clouds. The IPA is, therefore, not able to take into account the 3D effect of clouds adequately and fully three-dimensional calculations are inevitable for the purpose of our present study.

\section{Discussion}

In the previous section we presented how the $3 \mathrm{D}$ effect influences the radiation field in the cloudy atmosphere. We discuss below on longwave radiation budget of the earth in the presence of broken clouds.

The cooling or heating rate inside a cloud can be described in terms of the flux divergence in the cloud. The volume-averaged flux divergence is

$$
\operatorname{div} \mathbf{F}=\frac{\int_{V}(\boldsymbol{\nabla} \cdot \mathbf{F}) d V}{V}=\frac{\int_{S} \mathbf{F} d S}{V},
$$

where $V$ denotes the total volume of the cloud and $S$ the total surface area. In the limit that the cloud is extended infinitely in a horizontal plane, Eq. (24) leads to

$$
\operatorname{div} \mathbf{F}=\frac{\int_{S} \mathbf{F} d S}{A H} \approx \frac{F^{\uparrow} A-\left(\pi I_{\mathrm{inc}}-F^{\downarrow}\right) A}{A H},
$$

where $A$ is the horizontal surface area at the cloud top or base. Equation (25) shows that the mean heating rate inside an isothermal and nonscattering cloud is given by

$$
-\operatorname{div} \mathbf{F}=-\frac{F^{\uparrow}-\left(\pi I_{\mathrm{inc}}-F^{\downarrow}\right)}{H} .
$$

With Eqs. (4)-(7) and (14), Eq. (26) leads to

$$
-\operatorname{div} \mathbf{F}=\pi\left(I_{\mathrm{inc}}-2 S_{c}\right) \frac{1-2 \Lambda}{H} .
$$

Substituting $E_{3}\left(\tau_{\mathrm{PP}}\right)$ for $\Lambda$ as the plane-parallel value, $\operatorname{div} F_{\mathrm{PP}}$, we have 

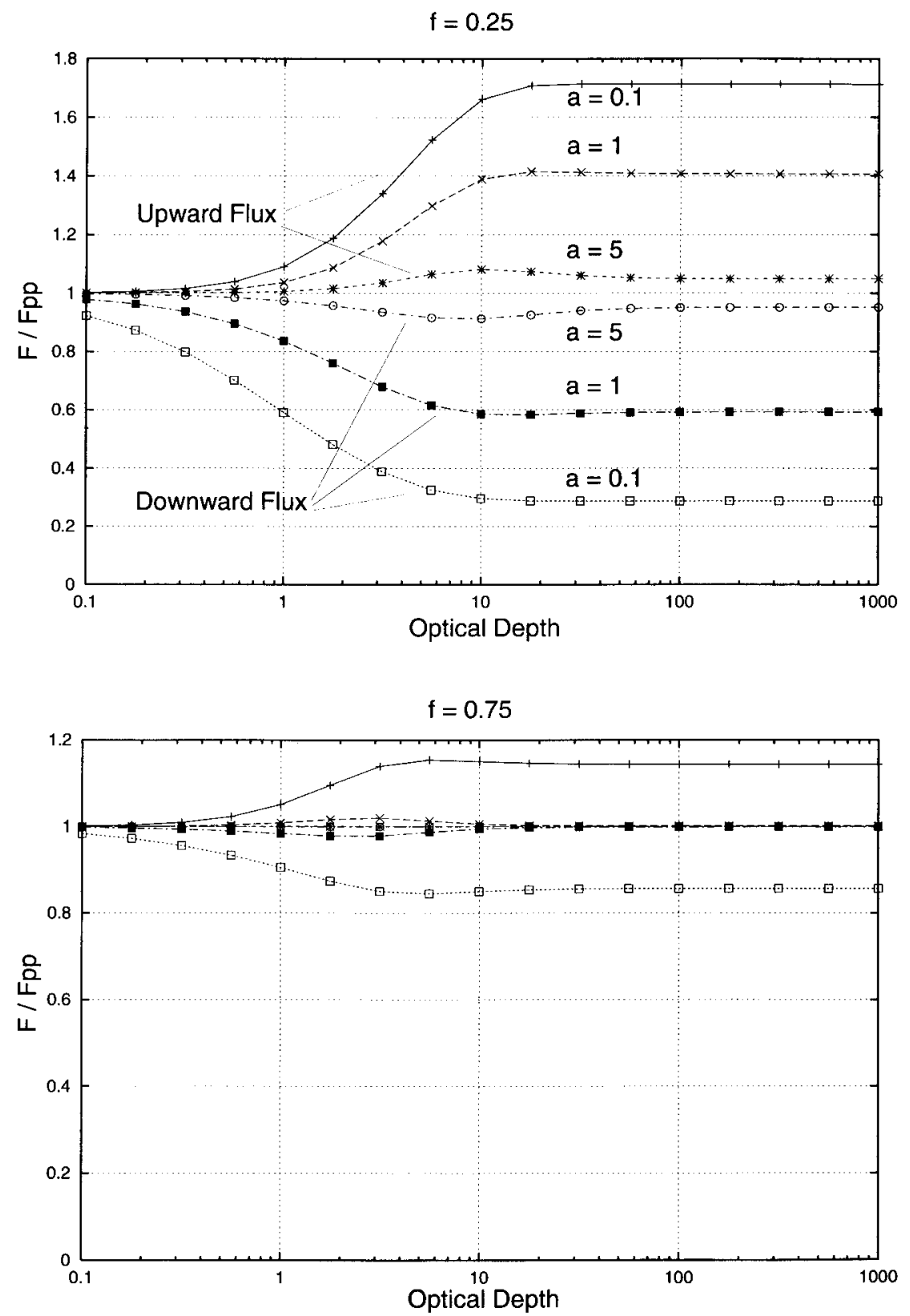

FIG. 7. The upward flux emergent through the cloud top and the downward flux through the base for the average plane-parallel model, i.e., $\tau_{\mathrm{PP}}=f \tau_{H}$.

$$
\frac{\operatorname{div} \mathbf{F}}{\operatorname{div} \mathbf{F}_{\mathrm{PP}}}=\frac{1-2 \Lambda}{1-2 E_{3}\left(\tau_{\mathrm{PP}}\right)}=f_{e},
$$

using Eq. (13). Therefore the longwave flux divergence inside an isothermal cloud normalized by the planeparallel value is equal to the effective cloud fraction.

The longwave radiative forcing of an isothermal and nonscattering cloud at its top is given by

$$
F_{\mathrm{rad}}=F^{\uparrow}-\pi I_{\mathrm{inc}},
$$

which leads to

$$
F_{\text {rad }}=\pi\left(S_{c}-I_{\text {inc }}\right)(1-2 \Lambda) .
$$

After the same procedure to derive Eq. (28), one has

$$
\frac{F_{\text {rad }}}{F_{\text {rad,PP }}}=\frac{1-2 \Lambda}{1-2 E_{3}\left(\tau_{\mathrm{PP}}\right)}=f_{e} .
$$

Thus the longwave forcing of an isothermal cloud normalized by the plane-parallel value as well accords with the effective cloud fraction.

Equations (28) and (31) indicate that $f_{e}$ shown in Figs. 5 and 8 is applicable also to examine the clouds' 

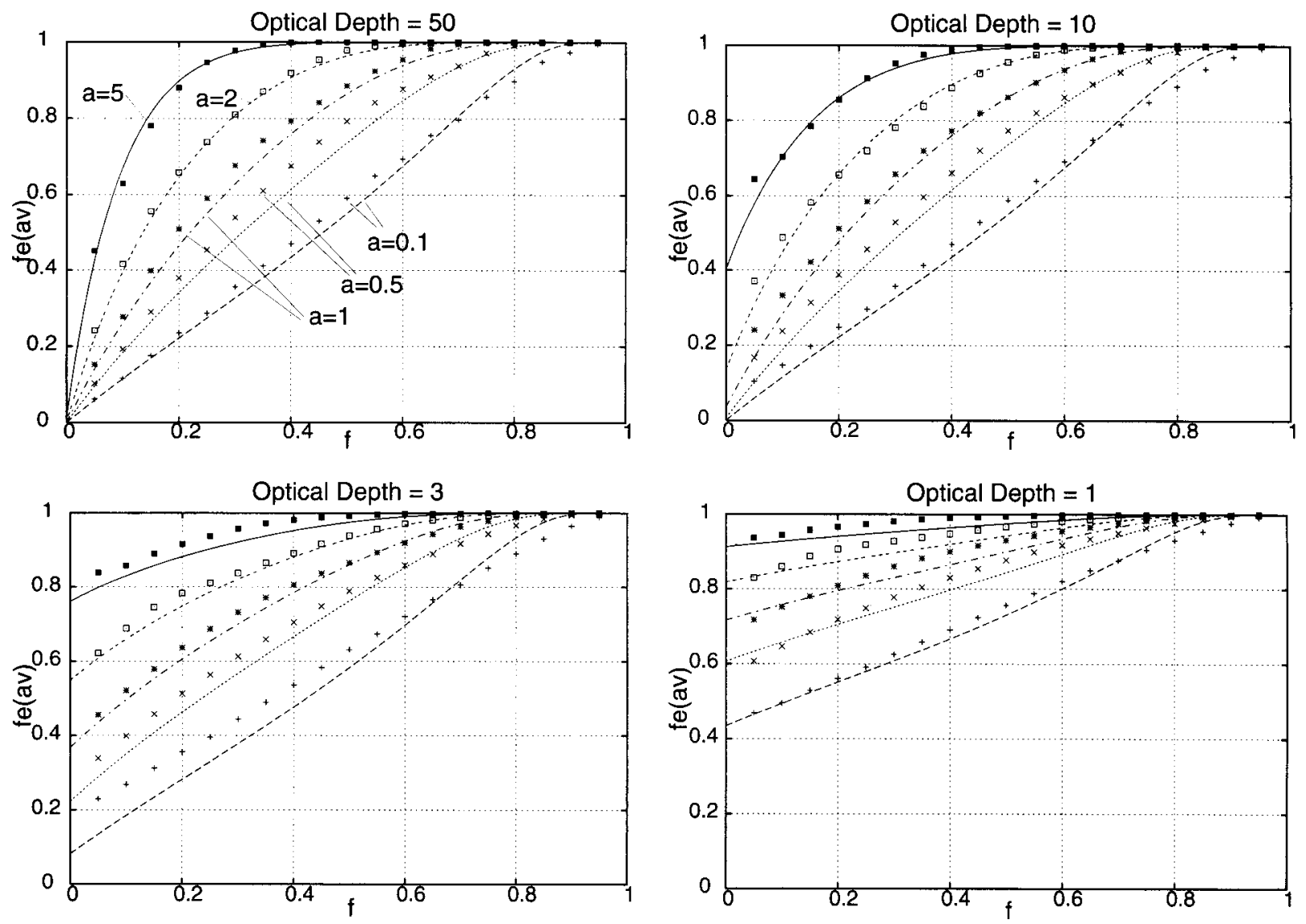

FIG. 8. Effective cloud fraction for $\tau_{H}=10,3,1$ as well as $\tau_{H}=50$ with the approximate formula given by Eq. (22) is shown (line). Symbols denote the exact solutions as in Fig. 3. The average plane-parallel model is used for the normalizing flux.

3D effects on the flux divergence and the radiative forcing for longwave radiation. This indicates that the planeparallel quantities, $(\boldsymbol{\nabla} \cdot \mathbf{F})_{\mathrm{PP}}$ and $F_{\text {rad,PP }}$, without any correction for the brokenness of cloud fields are overestimated by a factor of $f_{e}^{-1}$ from the true values. On the other hand, one finds that modified plane-parallel flux using the cloud fraction $f$ through Eqs. (17) and (18) would yield the flux divergence of $f(\boldsymbol{\nabla} \cdot \mathbf{F})_{\mathrm{PP}}$ and the radiative forcing of $f F_{\text {rad,PP }}$, replacing $f_{e}$ with $f$ in Eqs. (28) and (31). In this case, the flux divergence and the radiative forcing for longwave radiation are underestimated by a factor of $f / f_{e}$ since $f$ is always smaller than $f_{e}$. The errors can cause substantial misestimation in the radiation budget of the cloudy atmosphere in any case when the $3 \mathrm{D}$ effect of clouds is not taken into account adequately.

In this work we assume a simple cloud model of a cloud array made of identical cuboids, which is not the case in actual cloud fields. Several authors have done statistical analysis of the cloud size distributions employing Landsat Multispectral Scanner (MSS) and Thematic Mapper (TM) data (e.g., Wielicki and Welch 1986; Cahalan and Joseph 1989; Davis et al. 1997; and references therein). Wielicki and Welch (1986) investi- gated the cloud size distribution in MSS images, and found that the cloud size distributions show encouraging similarities among the cloud fields examined, compiling as well some previous studies based on aircraft photographs by Plank (1969) and Hozumi et al. (1982). Cahalan and Joseph (1989) also derived the cloud size distribution using TM data. They reported that the size distribution as a function of $\log (\sqrt{\text { cloud area }})$, or cloud effective diameter, is excellently approximated by a double power-law fit with the power of -0.89 for smaller scales than $\sim 2 \mathrm{~km}$ and of -2.76 for larger scales.

Davis et al. (1997) obtained the energy spectrum of the radiance field of clouds in a TM image, showing the power-law distribution in the Fourier space with two scale breaks at $0.2-0.4$ and $\approx 20 \mathrm{~km}$. Although direct comparison is impossible between the energy spectrum of cloud fields and the size distribution for ensembles of individual clouds as described above, cloud fields seem to be characterized by power-law distributions in general over a wide range of size scales.

Straightforward application of the results in this work to cloud fields with power-law size distribution is difficult. The effective cloud fraction involves radiative transfer problems, which do not linearly response to 
cloud size distribution. Moreover, radiative interaction between clouds with different sizes should be taken into account. A more sophisticated 3D RT scheme is required to solve such problems.

\section{Summary and conclusions}

We summarize the conclusions in this paper as follows.

1) We investigate the effects of broken clouds on longwave radiation on the basis of a simple cloud model that consists of a cuboidal cloud array. In order to extend the study of HW82 to be applicable to semitransparent clouds as well as optically thick clouds, multistream radiative transfer is solved to obtain the exact solution of radiative flux passing through the broken clouds. Since we concentrate the present study on the geometrical effects of broken clouds to avoid uncertainties lying in optical properties of cloud water droplets and ice crystals, the calculated flux is normalized by plane-parallel flux.

2) We find that the empirical formula for the effective cloud fraction previously derived by HW82 systematically underestimates the exact solution. Another formula [Eq. (20)] is proposed to provide a better fit to the exact solution.

3) In contrast to HW82, who considered only blackbody clouds, we also examine the dependence of radiative flux and the effective cloud fraction on optical thickness. Two different models are considered for computing plane-parallel flux used for the normalization: the maximum model $\left(\tau_{\mathrm{PP}}=\tau_{H}\right)$ and the average model $\left(\tau_{\mathrm{PP}}=f \tau_{H}\right)$. Approximate formulas of the effective cloud fraction for general optical thickness are proposed in Eq. (22) for the maximum plane-parallel case and in Eq. (23) for the average plane-parallel case.

4) The 3D effect of clouds can affect the thermal structure in the atmosphere. Neglecting the 3D effect causes overestimation by $f_{e}$ of the longwave flux divergence and the longwave forcing, while it leads to underestimation by $f / f$ if the cloud fraction $f$ is used instead of $f_{e}$ for modifying plane-parallel flux.

The 3D effect of cloud for shortwave radiation, where the scattering dominates the absorption in radiative processes, arises in a somewhat different way. Our subsequent work will be dedicated to the shortwave version of the present study.

Acknowledgments. The authors greatly appreciate detailed comments of anonymous reviewers, which have improved the manuscript.

\section{REFERENCES}

Aida, M., 1977: Reflection of solar radiation from an array cumuli. J. Meteor. Soc. Japan, 55, 174-181.

Cahalan, R. F., and J. H. Joseph, 1989: Fractal statistics of cloud fields. Mon. Wea. Rev., 117, 261-272.

Davis, A., A. Marshak, R. Cahalan, and W. Wiscombe, 1997: The Landsat scale break in stratocumulus as a three-dimensional radiative transfer effect: Implications for cloud remote sensing. $J$. Atmos. Sci., 54, 241-260.

Ellingson, R. G., 1982: On the effects of cumulus dimensions on longwave irradiance and heating rates. J. Atmos. Sci., 39, 886896.

Gabriel, P. M., S.-C. Tsay, and G. L. Stephens, 1993: A FourierRiccati approach to radiative transfer. Part I: Foundations. $J$. Atmos. Sci., 50, 3125-3147.

Han, D., and R. G. Ellingson, 1999: Cumulus cloud formulations for longwave radiation calculations. J. Atmos. Sci., 56, 837-851.

Harshvardhan, and J. A. Weinman, 1982: Infrared radiative transfer through a regular array of cuboidal clouds. J. Atmos. Sci., 39, 431-439.

,-- , and R. Davis, 1981: Transport of infrared radiation in cuboidal clouds. J. Atmos. Sci., 38, 2500-2513.

Hozumi, K., T. Harimaya, and C. Magono, 1982: The size distribution of cumulus clouds as a function of cloud amount. J. Meteor. Soc. Japan, 60, 691-699.

Killen, R., and R. G. Ellingson, 1994: The effect of shape and spatial distribution of cumulus clouds on longwave irradiance. J. Atmos. Sci., 51, 2123-2136.

Lewis, E. E., and W. F. Miller, 1984: Computational Methods of Neutron Transport. John Wiley and Sons, $401 \mathrm{pp}$.

Marshak, A., A. Davis., W. Wiscombe, and R. Cahalan, 1995: Radiative smoothing in fractal clouds. J. Geophys. Res., 100, 26 247-26 261.

Plank, V. G., 1969: The size distribution of cumulus clouds in representative Florida populations. J. Appl. Meteor., 8, 46-67.

Roewe, D., and K.-N. Liou, 1978: Influence of cirrus clouds on the infrared cooling rate in the troposphere and lower stratosphere. J. Appl. Meteor., 17, 92-107.

Takara, E., and R. G. Ellingson, 1996: Scattering effects on longwave fluxes in broken cloud fields. J. Atmos. Sci., 53, 1464-1476.

Wielicki, B. A., and R. M. Welch, 1986: Cumulus cloud properties derived using Landsat satellite data. J. Climate Appl. Meteor., 25, 261-276. 